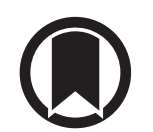

CrossMark

\section{Attitudes of patients with chronic breathlessness towards treatment with opioids}

\author{
To the Editor:
}

Breathlessness is the most common symptom in advanced chronic lung disease or chronic heart failure (CHF) [1]. Opioids are recommended for palliative treatment of breathlessness persisting despite optimal pharmacological and non-pharmacological treatment [2, 3]. However, physicians don't always consider opioids for chronic breathlessness $[4,5]$ and experience barriers when considering opioids, such as resistance of patients [6]. This can limit effective palliative treatment. Qualitative studies in patients with COPD and CHF revealed fear of dependence and fear of imminent death as the most important barriers to opioid use. The reason to start treatment was to do as much as possible [7-9]. These qualitative studies were only conducted in small patient populations. Therefore, our aims were to assess the willingness of patients with chronic lung disease or CHF to use opioids for breathlessness, irrespective of a current indication for opioid treatment; and to assess their barriers towards opioid use and reasons to use opioids. Finally, we aimed to compare willingness differences according to sex, age, educational level, diagnosis and breathlessness severity.

An exploratory convenience sample of 175 patients referred for a baseline assessment prior to pulmonary or heart failure rehabilitation was recruited, independent of their level of breathlessness. Patients were excluded if they could not read or write, had not mastered Dutch, were aged $<18$ years or were unable to give informed consent. The Maastricht University Medical Centre (MUMC+, Maastricht, the Netherlands) medical ethical committee, reviewed the study protocol and concluded that the study didn't fall under the Medical Research Involving Human Subjects Act (METC 2018-0790). Patients completed a survey including demographic characteristics, educational level, previous and current opioid use (opioid, dosage and reason for prescription) and willingness to use opioids for breathlessness. Patients willing to use opioids and patients who had experience with opioids were asked to indicate their reasons to use opioids; patients unwilling to use opioids were asked to indicate their barriers. Patients who were indecisive were asked to indicate their barriers against and the reasons to use opioids. In addition, patients were invited to report reasons other than those predefined using a free-text field. The predefined reasons were selected based on previous research [7-9], expert opinion of the project group and experience from patient inclusion for an opioid trial (MORDYC) [10]. Disease characteristics (diagnosis, disease history, lung function, 6-min walk distance (6MWD) [11]) and breathlessness severity (modified Medical Research Council (mMRC) scale) [12] were recorded using chart review.

Data were described using mean \pm SD or median (interquartile range (IQR)) for continuous variables and $n$ (\%) for categorical variables. We compared the willingness to use opioids between sexes, age ( $<65$ years or $\geqslant 65$ years), educational level (lower education, defined as having finished secondary vocational education versus higher education, defined as having finished at least higher general secondary education) [13], diagnosis and mMRC score ( $<2$ or $\geqslant 2$ points) using Chi-squared test or Fisher-Freeman-Halton test, as appropriate.

Between November 2018 and May 2019, 237 patients were eligible and 175 patients (50\% male), median age 65 years (IQR 57-70 years), completed the survey (response rate $74 \%$ ). Patients were diagnosed with

@ERSpublications

Attitudes of patients towards opioid treatment for chronic breathlessness are mixed, with $37 \%$ of patients willing to use opioids, $25 \%$ unwilling and $38 \%$ of patients indecisive. Physicians are an important source of information for these patients. http://bit.ly/2pvNtLJ

Cite this article as: Verberkt CA, van den Beuken-van Everdingen MHJ, Wouters EFM, et al. Attitudes of patients with chronic breathlessness towards treatment with opioids. Eur Respir J 2020; 55: 1901752 [https://doi.org/10.1183/13993003.01752-2019]. 
TABLE 1 Attitudes of patients with chronic breathlessness to opioid treatment

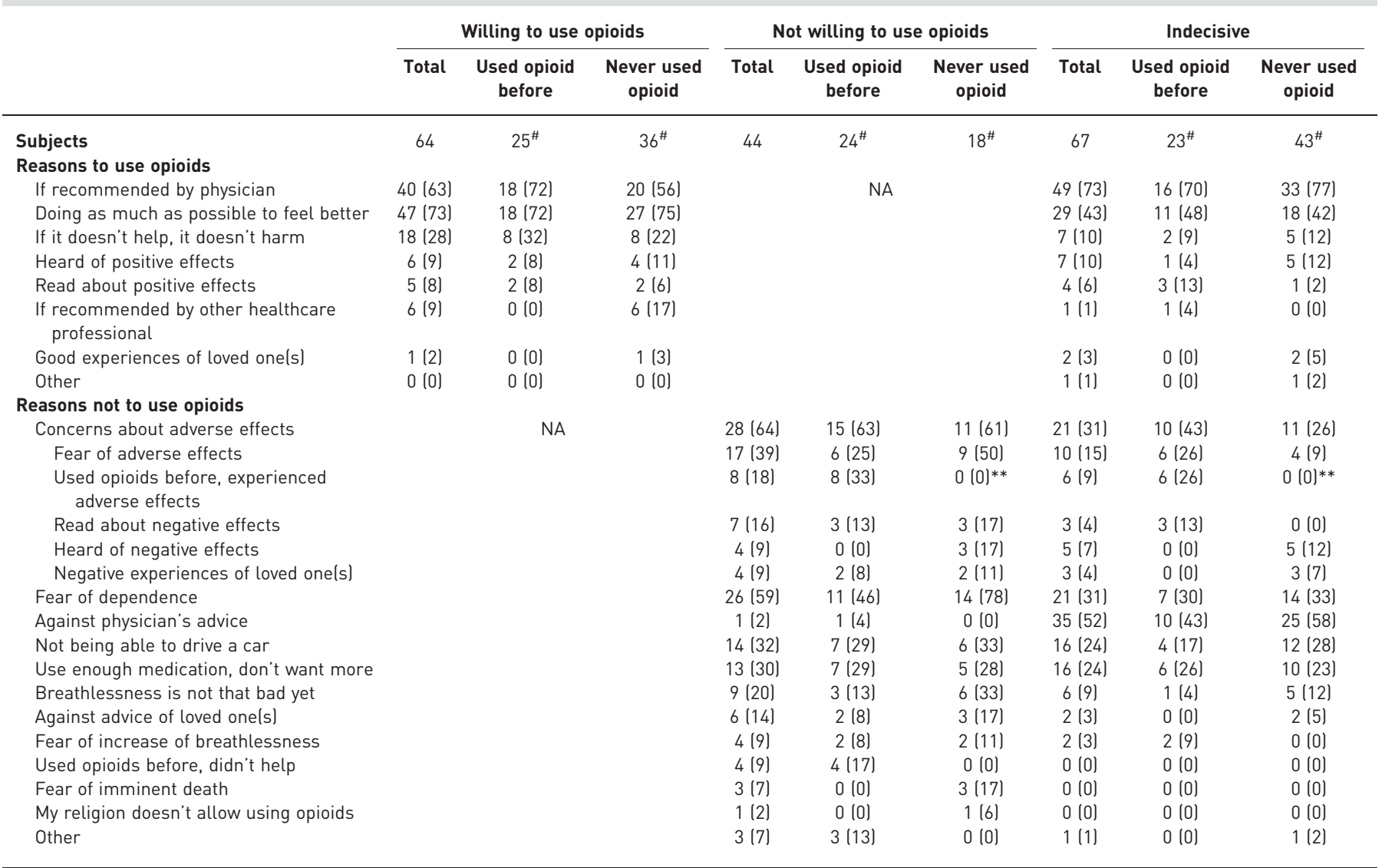

Data are presented as $\mathrm{n}$ or $\mathrm{n}(\%)$. NA: not applicable. " : six patients didn't know if they had used opioids before and are therefore excluded from this stratified analysis. ${ }^{* *}$ : $p<0.01$ for difference between patients who had used opioids before and patients who had never used opioids.

$\operatorname{COPD}(\mathrm{n}=124,71 \%), \operatorname{CHF}(\mathrm{n}=18,10 \%)$, asthma $(\mathrm{n}=17,10 \%)$, interstitial lung disease $(\mathrm{n}=8,5 \%)$, COPDasthma overlap syndrome $(n=3,2 \%)$, pulmonary hypertension $(n=3,2 \%)$ and other $(n=2,1 \%)$. Median mMRC score was 2.5 points (IQR 2-3 points), 6MWD was $393 \pm 107 \mathrm{~m}$ and 141 (81\%) patients completed lower education only. Nonresponders were $45 \%$ male and aged median 66 years (IQR 59-71 years) (both p $>0.05$ compared to responders).

$72(41 \%)$ out of 175 patients previously used one or more different opioids (49 (68\%) for pain, 10 (14\%) for breathlessness, eight (11\%) for both pain and breathlessness, five (7\%) for drug dependence and six (8\%) for unknown reasons). Opioids used were morphine $(n=45,63 \%)$, oxycodone $(n=30,42 \%)$, fentanyl $(n=7,10 \%)$, methadone $(n=6,8 \%)$ and buprenorphine $(n=3,4 \%)$. At the time of the survey, $14(8 \%)$ out of 175 patients used an opioid (seven (50\%) for pain, five (36\%) for breathlessness, one (7\%) for both pain and breathlessness and one (7\%) for drug dependence). Six (43\%) patients used morphine, four (29\%) patients used fentanyl, four (29\%) patients used oxycodone and one (7\%) patient used methadone. Median daily morphine equivalent dose was $27.5 \mathrm{mg}$ (IQR $16.25-30.00 \mathrm{mg}$ ) with an outlier of $480 \mathrm{mg}$ (dependence).

In total, $64(37 \%)$ patients were willing to use opioids for breathlessness, 44 (25\%) patients were unwilling and $67(38 \%)$ patients were indecisive. Patients unwilling to use opioids were older compared to patients willing to use opioids and indecisive patients (66 versus 63 and 62 years, respectively; $p<0.01$ ). Sex, educational level, diagnosis, mMRC score and previous opioid use were comparable between groups ( $\mathrm{p}=0.79, \mathrm{p}=0.06, \mathrm{p}=0.42, \mathrm{p}=0.06$ and $\mathrm{p}=0.07$, respectively).

Main reasons to use opioids were on physician's recommendation (89 (68\%) out of 131) and doing as much as possible to feel better $(79(60 \%)$ out of 131$)$. Main reasons not to use opioids were concerns about adverse effects (49 (44\%) out of 111) and fear of dependence (47 (42\%) out of 111). Differences existed between decisive patients and indecisive patients (table 1). Free-text considerations were not being familiar with opioids, fear of getting high and fear of weight increase. 
This study assessed attitudes towards opioid treatment in patients with chronic breathlessness with or without an indication for palliative treatment with opioids. The results showed that attitudes towards opioids are mixed, with only a quarter of the patients unwilling to use opioids. These results do not correspond with physicians' assumptions, who indicate that they do not prescribe opioids because patients are resistant [6]. Three-quarters of the indecisive patients indicated that they rely on physicians' positive advice and one-third indicated that they rely on negative advice. So the physician is an important source of information, and open and honest communication is important [8]. Since physicians state that they are insecure in prescribing opioids because of a lack of knowledge $[6,8]$, more emphasis should be on educating physicians about when and how to treat breathlessness with opioids.

The main barriers against and reasons to use opioids mentioned in this study were consistent with previous studies [7-9]. Concerns related to adverse effects and fear of dependence are the main reasons not to use opioids or to be indecisive. Qualitative studies show that patients experience that small improvements in breathlessness can have a big impact on the quality of life [9], which fits with the attitude of being willing to do as much as possible.

This study's results have also shown that there are as many opinions as people, which also applies to the way patients gather information to form this opinion. Therefore, a proper provision of information for patients using different channels is necessary.

Low-dose opioids appear to be effective and safe in patients with severe chronic breathlessness [14-16]. However, adverse effects might occur [15]. Therefore, physicians and patients should discuss benefits and possible harms when considering low-dose opioids.

A limitation of this study is that the convenience sample consisted of only 175 patients referred to one centre. Another limitation is that not all included patients had an indication for opioid treatment. However, there was no difference in attitude between patients with mild $(\mathrm{mMRC}<2)$ and severe breathlessness, indicating that forming an opinion about opioids is irrespective of breathlessness severity and therefore information should be suitable and accessible to all patients with chronic breathlessness. Nevertheless, opioids should be reserved for patients with chronic breathlessness, persisting despite optimal pharmacological and non-pharmacological treatment $[17,18]$.

Cornelia A. Verberkt $\oplus^{1}$, Marieke H.J. van den Beuken-van Everdingen $\oplus^{2}$, Emiel F.M. Wouters ${ }^{3,4}$ and Daisy J.A. Janssen ${ }^{1,3}$

${ }^{1}$ Dept of Health Services Research, CAPHRI School for Public Health and Primary Care, Faculty of Health Medicine and Life Sciences, Maastricht University, Maastricht, The Netherlands. ${ }^{2}$ Centre of Expertise for Palliative Care, Maastricht University Medical Centre (MUMC+), Maastricht, The Netherlands. ${ }^{3}$ Dept of Research and Development, CIRO, Horn, The Netherlands. ${ }^{4}$ Dept of Respiratory Medicine, Maastricht University Medical Centre (MUMC+), Maastricht, The Netherlands.

Correspondence: Cornelia A. Verberkt, P.O. Box 6166200 MD Maastricht, The Netherlands. E-mail: c.vandenberg@ maastrichtuniversity.nl

Received: 5 Sept 2019 | Accepted after revision: 24 Oct 2019

Acknowledgements: We would like to thank Miranda Coenjaerds (CIRO, Horn, the Netherlands) for contributing to the data collection. Furthermore, we would like to thank Liesbeth van Hoef and Wendy Engering (Centre of Expertise for Palliative Care, Maastricht University Medical Centre (MUMC+), Maastricht, the Netherlands) for entering the data.

Conflict of interest: C.A. Verberkt reports grants from ZonMW (836031012), the Hague, during the conduct of the study. M.H.J. van den Beuken-van Everdingen reports grants from ZonMW (836031012), during the conduct of the study. E.F.M Wouters reports personal fees for advisory board work from Nycomed and Boehringer, grants from AstraZeneca and GSK, personal fees for lectures from AstraZeneca, GSK, Novartis and Chiesi, outside the submitted work. D.J.A. Janssen reports grants from The Netherlands Organisation for Health Research and Development (ZonMW; grant 836031012), the Hague, the Netherlands, during the conduct of the study; personal fees for lectures from Boehringer Ingelheim, Novartis and AstraZeneca, outside the submitted work.

Support statement: This project was funded by the Netherlands Organisation for Health Research and Development (ZonMW), the Hague, the Netherlands (grant number 836031012). Funding information for this article has been deposited with the Crossref Funder Registry.

\section{References}

1 Janssen DJ, Spruit MA, Wouters EF, et al. Daily symptom burden in end-stage chronic organ failure: a systematic review. Palliat Med 2008; 22: 938-948.

2 Marciniuk DD, Goodridge D, Hernandez P, et al. Managing dyspnea in patients with advanced chronic obstructive pulmonary disease: a Canadian Thoracic Society clinical practice guideline. Can Respir J 2011; 18: 69-78. 
3 Lanken PN, Terry PB, Delisser HM, et al. An official American Thoracic Society clinical policy statement: palliative care for patients with respiratory diseases and critical illnesses. Am J Respir Crit Care Med 2008; 177: 912-927.

4 Carette $\mathrm{H}$, Zysman M, Morelot-Panzini C, et al. Prevalence and management of chronic breathlessness in COPD in a tertiary care center. BMC Pulm Med 2019; 19: 95.

5 Ahmadi Z, Sandberg J, Shannon-Honson A, et al. Is chronic breathlessness less recognised and treated compared with chronic pain? A case-based randomised controlled trial. Eur Respir J 2018; 52: 1800887.

6 Janssen DJ, de Hosson S, bij de Vaate E, et al. Attitudes toward opioids for refractory dyspnea in COPD among Dutch chest physicians. Chron Respir Dis 2015; 12: 85-92.

7 Oxberry SG, Jones L, Clark AL, et al. Attitudes to morphine in chronic heart failure patients. Postgrad Med J 2012; 88: 515-521.

8 Rocker G, Young J, Donahue M, et al. Perspectives of patients, family caregivers and physicians about the use of opioids for refractory dyspnea in advanced chronic obstructive pulmonary disease. CMAJ 2012; 184: E497-E504.

9 Rocker GM, Simpson AC, Young J, et al. Opioid therapy for refractory dyspnea in patients with advanced chronic obstructive pulmonary disease: patients' experiences and outcomes. CMAJ Open 2013; 1: E27-E36.

10 Verberkt CA, van den Beuken-van Everdingen MH, Franssen FM, et al. A randomized controlled trial on the benefits and respiratory adverse effects of morphine for refractory dyspnea in patients with COPD: protocol of the MORDYC study. Contemp Clin Trials 2016; 47: 228-234.

11 Spruit MA, Polkey MI, Celli B, et al. Predicting outcomes from 6-minute walk distance in chronic obstructive pulmonary disease. J Am Med Dir Assoc 2012; 13: 291-297.

12 Bestall JC, Paul EA, Garrod R, et al. Usefulness of the Medical Research Council (MRC) dyspnoea scale as a measure of disability in patients with chronic obstructive pulmonary disease. Thorax 1999; 54: 581-586.

13 Verhage F. Intelligentie en Leeftijd: Onderzoek bij Nederlanders van Twaalf tot Zevenenzeventig Jaar. [Intelligence and Age: Research among Dutch People aged Twelve to Seventy-seven Years]. Assen, van Gorcum, 1964.

14 Ekström MP, Bornefalk-Hermansson A, Abernethy AP, et al. Safety of benzodiazepines and opioids in very severe respiratory disease: national prospective study. BMJ 2014; 348: g445.

15 Ekström M, Bajwah S, Bland JM, et al. One evidence base; three stories: do opioids relieve chronic breathlessness? Thorax 2018; 73: 88-90.

16 Verberkt CA, van den Beuken-van Everdingen MHJ, Schols J, et al. Respiratory adverse effects of opioids for breathlessness: a systematic review and meta-analysis. Eur Respir J 2017; 50: 1701153.

17 Currow D, Louw S, McCloud P, et al. Regular, sustained-release morphine for chronic breathlessness: a multicentre, double-blind, randomised, placebo-controlled trial. Thorax 2020; 75: 50-56.

18 Johnson MJ, Bland JM, Oxberry SG, et al. Opioids for chronic refractory breathlessness: patient predictors of beneficial response. Eur Respir J 2013; 42: 758-766. 